\title{
Compact endomorphisms of Banach algebras of infinitely differentiable functions
}

\author{
Joel F. Feinstein and Herbert Kamowitz
}

November 9, 2018

\begin{abstract}
Let $\left(M_{n}\right)$ be a sequence of positive numbers satisfying $M_{0}=1$ and $\frac{M_{n+m}}{M_{n} M_{m}} \geq\left(\begin{array}{c}n+m \\ m\end{array}\right)$ for all non-negative integers $m, n$. We let

$$
D([0,1], M)=\left\{f \in C^{\infty}([0,1]):\|f\|_{D}=\sum_{n=0}^{\infty} \frac{\left\|f^{(n)}\right\|_{\infty}}{M_{n}}<\infty\right\} .
$$

With pointwise addition and multiplication, $D([0,1], M)$ is a unital commutative semisimple Banach algebra. If $\lim _{n \rightarrow \infty}\left(n ! / M_{n}\right)^{1 / n}=0$, then the maximal ideal space of the algebra is $[0,1]$ and every non-zero endomorphism $T$ has the form $T f(x)=f(\phi(x))$ for some selfmap $\phi$ of the unit interval. Previously we have shown for a wide class of $\phi$ mapping the unit interval to itself that if $\left\|\phi^{\prime}\right\|_{\infty}<1$ then $\phi$ induces a compact endomorphism. Here we investigate the extent to which this condition is necessary, and we determine the spectra of all compact endomorphisms of $D([0,1], M)$. We also simplify and strengthen some of our earlier results on general endomorphisms of $D([0,1], M)$.
\end{abstract}

\section{Introduction}

In two previous papers [5] and [7] we studied endomorphisms of certain algebras of infinitely differentiable functions. In this note, we continue our study, concentrating on algebras of functions on compact intervals in the real line. Let $\left(M_{n}\right)$ be a sequence of positive numbers satisfying $M_{0}=1$ and $\frac{M_{n+m}}{M_{n} M_{m}} \geq\left(\begin{array}{c}n+m \\ n\end{array}\right), m, n$, non-negative integers. If $X$ is a compact interval of positive length we let

$$
D(X, M)=\left\{f \in C^{\infty}(X):\|f\|_{D}=\sum_{n=0}^{\infty} \frac{\left\|f^{(n)}\right\|_{\infty}}{M_{n}}<\infty\right\} .
$$


With pointwise addition and multiplication, $D(X, M)$ is a unital commutative semisimple Banach algebra. The maximal ideal space of $D(X, M)$ depends on $\left(M_{n}\right)$. In particular, it was shown in [4] (see also [3] Theorem 4.4.16) that if $\left(M_{n}\right)$ is a non-analytic sequence, i.e. $\lim _{n \rightarrow \infty}\left(\frac{n !}{M_{n}}\right)^{1 / n}=0$, then the maximal ideal space of $D(X, M)$ is precisely $X$.

A stronger condition on the sequence $\left(M_{n}\right)$ is that $\sum \frac{M_{n}}{M_{n+1}}<\infty$. In this case the sequence $\left(M_{n}\right)$ is non-analytic and, in addition, $D(X, M)$ is regular ([3], Theorem 4.4.22). Such a weight sequence will be called nonquasi-analytic.

When the weight $\left(M_{n}\right)$ is non-analytic, every non-zero endomorphism $T$ of the algebra $D(X, M)$ has the form $T f(x)=f(\phi(x))$ for some $\phi: X \rightarrow X$. This leads us to ask which $\phi: X \rightarrow X$ induce endomorphisms of $D(X, M)$ ? That is, which $\phi$ satisfy $f \circ \phi \in D(X, M)$ whenever $f \in D(X, M)$ ? We observe that since $f(x)=x$ is in $D(X, M)$ for all $X$ and $\left(M_{n}\right)$, for $\phi$ to induce an endomorphism it is necessary that $\phi \in D(X, M)$.

We sometimes require an additional condition on an infinitely differentiable selfmap $\phi$ of the interval $X$ : we say that $\phi$ is analytic if

$$
\sup _{k}\left(\frac{\left\|\phi^{(k)}\right\|_{\infty}}{k !}\right)^{1 / k}<\infty .
$$

In [7] the following theorems were proved concerning analytic selfmaps $\phi$ of $[0,1]$.

Theorem A: Let $\left(M_{n}\right)$ be a non-analytic weight sequence and let $\phi$ be an analytic selfmap of $[0,1]$ with $\left\|\phi^{\prime}\right\|_{\infty}<1$. Then $\phi$ induces an endomorphism of $D([0,1], M)$, and this endomorphism is compact.

Theorem $\mathbf{A}^{\prime}$ : Let $\left(M_{n}\right)$ be a non-analytic weight sequence such that there is a constant $B>0$ with $\frac{M_{m}}{m !} \frac{n !}{M_{n}} \leq \frac{B}{m^{n-m}}$. Let $\phi$ be an infinitely differentiable selfmap of $[0,1]$ such that $\left\|\phi^{\prime}\right\|_{\infty} \leq 1$, and $\left(\frac{\left\|\phi^{(k)}\right\|_{\infty}}{k !}\right)$ is bounded. Then $\phi$ induces an endomorphism of $D([0,1], M)$.

Theorem B: Suppose that $\left(M_{n}\right)$ is a non-analytic weight sequence and that $\phi$ is an analytic selfmap of $[0,1]$. If $\phi$ induces an endomorphism of $D([0,1], M)$, then $\left\|\phi^{\prime}\right\|_{\infty} \leq 1$.

In Part I of this paper we investigate when a converse to the compactness part of Theorem $\mathrm{A}$ is true for $D([0,1], M)$; namely, if $\phi$ induces a compact endomorphism of $D([0,1], M)$ must $\left\|\phi^{\prime}\right\|_{\infty}<1$ ? Along the way we 
prove a stronger version of Theorem B, eliminating the analyticity assumption on $\phi$ and removing the need for the technical calculations used in the original proof. In Part II, we determine the spectra of compact endomorphisms of $D([0,1], M)$. Part III contains results on general endomorphisms of $D([0,1], M)$. In particular, we simplify and weaken slightly the growth condition on the sequence $M$ from Theorem $\mathrm{A}^{\prime}$ and we investigate the extent to which this condition is necessary.

We would like to thank the referee for some very helpful comments.

\section{Part I. Compact endomorphisms}

We begin by recalling some facts about endomorphisms. Let $B$ be a commutative, unital semisimple Banach algebra with a connected maximal ideal space $X$, and suppose that $T$ is a non-zero endomorphism of $B$. Then there exists a $w *$-continuous function $\phi: X \rightarrow X$ with $\widehat{T f}(x)=\hat{f}(\phi(x))$ for $f \in B$ and $x \in X$. We let $\phi_{n}$ denote the $n^{\text {th }}$ iterate of $\phi$. If the endomorphism $T$ is compact, then it was shown in [6] that $\bigcap_{n=0}^{\infty} \phi_{n}(X)=\left\{x_{0}\right\}$ where $x_{0} \in X$ is a fixed point of $\phi$ which clearly must be unique. In view of this the following result is not surprising.

Theorem 1.1: Suppose that $X$ is a compact interval of positive length and $\left(M_{n}\right)$ is a weight sequence. Suppose that $\phi$ induces a compact endomorphism of $D(X, M)$. If $x_{0}$ is the fixed point of $\phi$, then $\left|\phi^{\prime}\left(x_{0}\right)\right|<1$.

Proof: Suppose, for a contradiction, that $\left|\phi^{\prime}\left(x_{0}\right)\right| \geq 1$. Then $\left\|\phi_{n}\right\|_{D} \geq$ $\frac{\left|\phi_{n}^{\prime}\left(x_{0}\right)\right|}{M_{1}}=\frac{\left(\left|\phi^{\prime}\left(x_{0}\right)\right|\right)^{n}}{M_{1}} \geq \frac{1}{M_{1}}$. It follows that the sequence of iterates $\phi_{n}$ has a subsequence $\phi_{n_{k}}$ for which there is a $\delta>0$ with $\frac{\left\|\phi_{n_{k}+1}\right\|_{D}}{\left\|\phi_{n_{k}}\right\|_{D}} \geq \delta$. For this subsequence let $f_{k}=\frac{\phi_{n_{k}}}{\left\|\phi_{n_{k}}\right\|_{D}}$. Since $\left\|f_{k}\right\|_{D}=1$ and $\phi$ induces a compact endomorphism there exists $g \in D(X, M)$ and a subsequence $\left\{f_{k_{j}}\right\}$ with $f_{k_{j}}(\phi) \rightarrow g$ in norm. With no loss of generality we call the subsequence $\left\{f_{k}\right\}$. Then $f_{k}(\phi)=\frac{\phi_{n_{k}+1}}{\left\|\phi_{n_{k}}\right\|_{D}} \rightarrow g$ in norm. Since $\bigcap_{n=0}^{\infty} \phi_{n}(X)=\left\{x_{0}\right\}$, we have $\lim _{n \rightarrow \infty} \phi_{n}(x)=x_{0}$ for each $x \in X$. Now

$$
g\left(x_{0}\right)=\lim _{k \rightarrow \infty} f_{k}\left(\phi\left(x_{0}\right)\right)=\lim _{k \rightarrow \infty} \frac{\phi_{n_{k}+1}\left(x_{0}\right)}{\left\|\phi_{n_{k}}\right\|_{D}}=\lim _{k \rightarrow \infty} \frac{x_{0}}{\left\|\phi_{n_{k}}\right\|_{D}} .
$$

Suppose, at first, that $x_{0} \neq 0$. Then $\lim _{k \rightarrow \infty} \frac{1}{\left\|\phi_{n_{k}}\right\|_{D}}=\frac{g\left(x_{0}\right)}{x_{0}}$. Hence for each 
$x \in X$,

$$
g(x)=\lim _{k \rightarrow \infty} f_{k}(\phi(x))=\lim _{k \rightarrow \infty} \frac{\phi_{n_{k}+1}(x)}{\left\|\phi_{n_{k}}\right\|_{D}}=g\left(x_{0}\right),
$$

whence $g$ is a constant function.

We consider in turn the two cases $g\left(x_{0}\right)=0$ and $g\left(x_{0}\right) \neq 0$.

(a) First, if $g\left(x_{0}\right)=0$, then $\lim _{k \rightarrow \infty} f_{k}(\phi)=0$ in norm. But $\left\|f_{k}(\phi)\right\|_{D}=$ $\frac{\left\|\phi_{n_{k}+1}\right\|_{D}}{\left\|\phi_{n_{k}}\right\|_{D}} \geq \delta>0$, a contradiction.

(b) Otherwise, $g\left(x_{0}\right) \neq 0$. Since $f_{k}(\phi) \rightarrow g$ in norm, we have $\left(f_{k} \circ\right.$ $\phi)^{\prime}(x) \rightarrow g^{\prime}(x)$ for all $x \in X$. Since $g$ is a constant function, $g^{\prime}(x)=0$ for all $x \in X$. However,

$$
\lim _{k \rightarrow \infty}\left|\left(f_{k} \circ \phi\right)^{\prime}\left(x_{0}\right)\right|=\lim _{k \rightarrow \infty}\left|\frac{\phi_{n_{k}+1}^{\prime}\left(x_{0}\right)}{\left\|\phi_{n_{k}}\right\|_{D}}\right| \geq \lim _{k \rightarrow \infty} \frac{1}{\left\|\phi_{n_{k}}\right\|_{D}}=\frac{g\left(x_{0}\right)}{x_{0}} \neq 0,
$$

again a contradiction. Hence, if $\left|\phi^{\prime}\left(x_{0}\right)\right| \geq 1$ and $x_{0} \neq 0$, then $\phi$ does not induce a compact endomorphism.

Finally, if $x_{0}=0$, we have

$$
g(x)=\lim _{k \rightarrow \infty} f_{k}(\phi(x))=\lim _{k \rightarrow \infty} \frac{\phi_{n_{k}+1}(x)}{\left\|\phi_{n_{k}}\right\|_{D}}=0
$$

for all $x$, and as in (a) above we again are led to a contradiction. Thus if $\left|\phi^{\prime}\left(x_{0}\right)\right| \geq 1$, then $\phi$ does not induce a compact endomorphism.

We remark that this result shows that if $\phi$ induces a compact endomorphism of $D([0,1], M)$, then some iterate $\phi_{n}$ satisfies $\left\|\phi_{n}^{\prime}\right\|_{\infty}<1$. We conjecture that $\phi$ itself satisfies $\left\|\phi^{\prime}\right\|_{\infty}<1$. Most of the remainder of this section is devoted to this question. Before we get on to the main focus of this section, however, we use Theorem 1.1 to produce a very short proof of a stronger version of Theorem B. In particular, observe that there is no restriction on the growth of the derivatives of $\phi$.

Theorem 1.2: Let $\left(M_{n}\right)$ be a non-analytic weight and let $\phi$ be a selfmap of $[0,1]$. If $\phi$ induces an endomorphism of $D([0,1], M)$, then $\left\|\phi^{\prime}\right\|_{\infty} \leq 1$.

Proof: Let $\phi$ induce an endomorphism of $D([0,1], M)$ and suppose that $\left|\phi^{\prime}\left(x_{0}\right)\right|>1$ for some $x_{0} \in[0,1]$. By continuity of $\phi^{\prime}$ it is no restriction to assume that $0<x_{0}<1$. It is easy to see that there exists a polynomial function $p:[0,1] \rightarrow[0,1]$ such that $p\left(\phi\left(x_{0}\right)\right)=x_{0}, p^{\prime}\left(\phi\left(x_{0}\right)\right)=\frac{1}{\left|\phi^{\prime}\left(x_{0}\right)\right|}$, and $\left\|p^{\prime}\right\|_{\infty}<1$. It then follows from Theorem A that $p$ induces a compact endomorphism of $D([0,1], M)$, whence $p \circ \phi$ also induces a compact endomorphism 
of the algebra. But $(p \circ \phi)\left(x_{0}\right)=x_{0}$, and $\left|(p \circ \phi)^{\prime}\left(x_{0}\right)\right|=\left|p^{\prime}\left(\phi\left(x_{0}\right)\right) \phi^{\prime}\left(x_{0}\right)\right|=1$. Since $x_{0}$ is a fixed point of $p \circ \phi$ we obtain a contradiction to Theorem 1.1. Thus $\left\|\phi^{\prime}\right\|_{\infty} \leq 1$.

We now continue with some results about homomorphisms rather than endomorphisms.

Let $X, Y$ be compact intervals in $\mathbf{R}$ of positive length, let $\phi$ be a map from $X$ to $Y$ and let $\left(M_{n}\right)$ be a non-analytic weight sequence. For $f$ in $D(Y, M)$ we look at $f \circ \phi$ and investigate whether it is in $D(X, M)$. In other words we ask whether $\phi$ induces a homomorphism from $D(Y, M)$ to $D(X, M)$. (Since these algebras are commutative, semi-simple Banach algebras, if this happens then the homomorphism must be continuous.) We also ask whether the homomorphism is compact.

We begin with some elementary observations, which follow easily from properties of the relevant restriction maps.

Observation 1 Suppose that $\phi: X \rightarrow Y$ induces a homomorphism from $D(Y, M)$ to $D(X, M)$. Let $X_{0}$ be a closed interval of positive length contained in $X$. Then $\phi_{\mid X_{0}}$ induces a homomorphism from $D(Y, M)$ to $D\left(X_{0}, M\right)$. Moreover, if the homomorphism induced by $\phi$ is compact, then so is the homomorphism induced by $\phi_{\mid X_{0}}$.

Observation 2: Let $Y_{0}$ be a closed interval in $Y$ such that $\phi(X) \subseteq Y_{0}$. If $\phi$ induces a homomorphism from $D\left(Y_{0}, M\right)$ to $D(X, M)$ then $\phi$ induces a homomorphism from $D(Y, M)$ to $D(X, M)$. Similarly, If $\phi$ induces a compact homomorphism from $D\left(Y_{0}, M\right)$ to $D(X, M)$ then $\phi$ induces a compact homomorphism from $D(Y, M)$ to $D(X, M)$.

We now prove the converse to the second of these observations under the additional assumption that the algebra is regular. We do not know whether this assumption is redundant.

Lemma 1.3 If $M$ is a non-quasi-analytic sequence (so that $D(Y, M)$ is regular) and $\phi(X)$ is a subset of the interior (relative to $Y$ ) of $Y_{0}$ then $\phi$ induces a homomorphism from $D\left(Y_{0}, M\right)$ to $D(X, M)$ if and only if $\phi$ induces a homomorphism from $D(Y, M)$ to $D(X, M)$, and the compactness of either homomorphism implies that of the other.

Proof: Suppose that $D(Y, M)$ is regular and that $\phi(X)$ is a subset of the interior (relative to $Y$ ) of $Y_{0}$. By regularity we may choose functions $g, h$ in $D(Y, M)$ such that $g$ is supported on the interior of $Y_{0}$, the support of $h$ does not meet $\phi(X)$ and $g+h=1$. Write $g_{1}=g_{\mid Y_{0}}$ and $h_{1}=h_{\mid Y_{0}}$. Suppose that $\phi$ induces a homomorphism from $D(Y, M)$ to $D(X, M)$. Let $f \in D\left(Y_{0}, M\right)$. We have $f=f g_{1}+f h_{1}$ and so (since $\left.h_{1} \circ \phi=0\right) f \circ \phi=\left(f g_{1}\right) \circ \phi$. Since $f g_{1}$ is supported on the interior of $Y_{0}$ we may extend it to obtain a function $F$ 
in $D(Y, M)$ with $F \circ \phi=\left(f g_{1}\right) \circ \phi=f \circ \phi$. By the assumption on $\phi, F \circ \phi \in$ $D(X, M)$, i.e. $f \circ \phi \in D(X, M)$. This shows that $\phi$ induces a homomorphism from $D\left(Y_{0}, M\right)$ to $D(X, M)$. Next suppose that the homomorphism induced from $D(Y, M)$ to $D(X, M)$ is compact. Let $f_{n}$ be a bounded sequence of functions in $D\left(Y_{0}, M\right)$. As above we have $f_{n}=f_{n} g_{1}+f_{n} h_{1}$ and $f_{n} \circ \phi=$ $\left(f_{n} g_{1}\right) \circ \phi$. Since the supports of the bounded sequence of functions $f_{n} g_{1}$ are contained in the interior of $Y_{0}$, these functions may be extended to functions $F_{n}$ in $D(Y, M)$ with the same norm and such that $F_{n} \circ \phi=\left(f_{n} g_{1}\right) \circ \phi=f_{n} \circ \phi$. Now, by the compactness assumption, $F_{n} \circ \phi$ has a convergent subsequence in $D(X, M)$, i.e. $f_{n} \circ \phi$ has a convergent subsequence. This shows that the homomorphism from $D\left(Y_{0}, M\right)$ to $D(X, M)$ induced by $\phi$ is compact.

Combining this with the second observation above gives the result.

The next lemma may appear to be obvious. Note, however, that our proof is only valid in the case where the algebra is regular.

Lemma 1.4: Suppose that $\left(M_{n}\right)$ is non-quasi-analytic and that $\phi^{\prime}$ is constantly 1 on $X$. Then $\phi$ does not induce a compact homomorphism from $D(Y, M)$ to $D(X, M)$.

Proof: We may assume (after translating) that $\phi(x)=x$. Choose a nondegenerate compact interval $X_{0}$ contained in the interior of $X$ and let $E_{1}$, $E_{2}$ be the closed subspaces of, respectively, $D(X, M), D(Y, M)$ consisting of those elements whose supports are subsets of $X_{0}$. Then these infinitedimensional Banach spaces are isometrically isomorphic to each other using the restriction to $E_{2}$ of the homomorphism induced by $\phi$. Since this map is clearly not compact, neither is the original homomorphism.

We are now ready to give our main result concerning necessary conditions on $\phi$ in order for $\phi$ to induce a compact endomorphism. Note that we do not assume that $\phi$ is analytic here.

Theorem 1.5: Let $\left(M_{n}\right)$ be a non-quasi-analytic algebra sequence and let $\phi$ be a map from $[0,1]$ to $[0,1]$. Suppose that $\phi$ induces a compact endomorphism of $D([0,1], M)$. Then for all $x \in(0,1)$ we have $\left|\phi^{\prime}(x)\right|<1$. Thus, if $\phi$ induces a compact endomorphism of $D([0,1], M)$ and $\left|\phi^{\prime}\right|$ peaks in $(0,1)$, then $\left\|\phi^{\prime}\right\|_{\infty}<1$.

Proof: Suppose that $\phi$ induces a compact endomorphism of $D([0,1], M)$. Since $\phi$ induces an endomorphism of $D([0,1], M)$ it follows from Theorem 1.2 that $\left\|\phi^{\prime}\right\|_{\infty} \leq 1$. Now suppose, for contradiction, that there is an $x_{1} \in(0,1)$ with $\left|\phi^{\prime}\left(x_{1}\right)\right|=1$. Composing with the map $t \mapsto(1-t)$ if necessary, we may assume that $\phi^{\prime}\left(x_{1}\right)=1$. Certainly $\phi\left(x_{1}\right) \in(0,1)$. Set

$$
\delta=\min \left\{x_{1}, 1-x_{1}, \phi\left(x_{1}\right), 1-\phi\left(x_{1}\right)\right\} .
$$


Set $a=x_{1}-\delta, b=x_{1}+\delta, c=x_{1}-\phi\left(x_{1}\right), X=[a, b]$ and $Y=[c, c+1]$. Define $\psi: X \rightarrow Y$ by $\psi(x)=\phi(x)+c$. From our assumptions, the observations above and obvious properties of translations, it is evident that $\psi$ must induce a compact homomorphism from $D(Y, M)$ to $D(X, M)$.

Set $Y_{0}=X$. Then $\psi(X) \subseteq Y_{0} \subseteq Y$. Note that $\psi\left(x_{1}\right)=x_{1}$ and $\psi^{\prime}\left(x_{1}\right)=1$ so, by Theorem 1.1, $\psi$ does not induce a compact endomorphism of $D(X, M)$ i.e. $\psi$ does not induce a compact homomorphism from $D\left(Y_{0}, M\right)$ to $D(X, M)$. In view of the observations above, this will contradict Lemma 1.3 provided that $\psi(X)$ is a subset of the interior of $Y_{0}$. The only way this could fail would be if $\psi^{\prime}$ was constantly equal to 1 on a non-degenerate compact subinterval of $X$, say $X_{0}$. This, however, would contradict the observations above, since, by Lemma 1.4, $\psi \mid X_{0}$ would not induce a compact homomorphism from $D(Y, M)$ to $D\left(X_{0}, M\right)$. The result now follows.

The only remaining case is when $\phi^{\prime}(0)=1$ or $\phi^{\prime}(1)=1$. Theorem 1.1 deals with this if the relevant point is a fixed point. Using the flip of the interval, the only remaining case is really when $\phi^{\prime}(1)=1$ and $\phi(1) \in$ $(0,1)$. That is, can we find a weight sequence $\left(M_{n}\right)$ and an analytic $\phi \in$ $D([0,1], M)$ with $\phi^{\prime}(1)=1$ and $0<\phi(1)<1$ such that $\phi$ induces a compact endomorphism of $D([0,1], M)$ ? This question is still open.

However, we have two partial results whose proofs we omit. First we can show that if $\phi$ is a quadratic function, and if $\phi$ induces a compact endomorphism of $D([0,1], M)$, then $\left\|\phi^{\prime}\right\|_{\infty}<1$. Secondly, the function $g$ defined by $g(z)=\sum_{n=0}^{\infty} \frac{z^{n}}{M_{n}}$ is an entire function. Using more sophisticated results on entire functions as developed in 2] and [8, we are also able to show that if the order of $g$ is less than $\frac{1}{2}$, or if the order of $g$ equals $\frac{1}{2}$ and the type of $g$ is finite, and if an analytic selfmap $\phi$ of $[0,1]$ induces a compact endomorphism of $D([0,1], M)$, then again $\left\|\phi^{\prime}\right\|_{\infty}<1$.

\section{Part II. Spectra of compact endomorphisms}

We next turn to the question of determining the spectra of compact endomorphisms of $D([0,1], M)$. We denote the spectrum of an operator $T$ by $\sigma(T)$. We also note that if $T$ is a non-zero endomorphism of $D([0,1], M)$, then $\lambda=1 \in \sigma(T)$ since $T 1=1$. Also, if $T$ is a compact endomorphism, then $0 \in \sigma(T)$.

We will show (in Theorems 2.4 and 2.5) that if $\phi$ induces a compact endomorphism $T$ of $D([0,1], M)$ and if $x_{0}$ is the fixed point of $\phi$, then $\sigma(T)=\left\{\left(\phi^{\prime}\left(x_{0}\right)\right)^{n}: n\right.$ is a positive integer $\} \cup\{0,1\}$, and each non-zero element of $\sigma(T)$ is an eigenvalue of multiplicity 1. First we require some 
lemmas.

Lemma 2.1: Let $\left(M_{n}\right)$ be a weight sequence and suppose that $\phi$ : $[0,1] \rightarrow[0,1]$ induces a compact endomorphism $T$ of $D([0,1], M)$ with $\phi\left(x_{0}\right)=x_{0}$. Then $\sigma(T) \supset\left\{\left(\phi^{\prime}\left(x_{0}\right)\right)^{n}: n\right.$ is a positive integer $\} \cup\{0,1\}$.

Proof: We first show that $\phi^{\prime}\left(x_{0}\right) \in \sigma(T)$. Indeed, no $f \in D([0,1], M)$ satisfies $\phi^{\prime}\left(x_{0}\right) f(x)-f(\phi(x))=x-x_{0}$. For, if this were not the case and $f$ satisfied $\phi^{\prime}\left(x_{0}\right) f(x)-f(\phi(x))=x-x_{0}$, then $\phi^{\prime}\left(x_{0}\right) f^{\prime}(x)-f^{\prime}(\phi(x)) \phi^{\prime}(x)=1$. But evaluating at $x=x_{0}$ gives a contradiction. Hence $\phi^{\prime}\left(x_{0}\right) \in \sigma(T)$.

Since $T$ is compact every non-zero element in $\sigma(T)$ is an eigenvalue of $T$. Clearly we may assume that $\phi^{\prime}\left(x_{0}\right) \neq 0$ and so, for some non-zero $g \in D([0,1], M), g(\phi(x))=\phi^{\prime}\left(x_{0}\right) g(x)$. Therefore, for each positive integer $n, g^{n}(\phi(x))=\phi^{\prime}\left(x_{0}\right)^{n} g^{n}(x)$, whence each $\phi^{\prime}\left(x_{0}\right)^{n} \in \sigma(T)$ for all positive integers $n$.

We remark that this lemma holds for non-compact endomorphisms as well. For, by successive differentiation, one can show that if $\phi\left(x_{0}\right)=x_{0}$, then for each positive integer $n$ there is no $f \in D([0,1], M)$ satisfying $\left(\phi^{\prime}\left(x_{0}\right)\right)^{n} f(x)-f(\phi(x))=\left(x-x_{0}\right)^{n}$. This again implies that $\left(\phi^{\prime}\left(x_{0}\right)\right)^{n} \in$ $\sigma(T)$ for all positive integers $n$.

Lemma 2.2: Suppose that $\left(M_{n}\right)$ is a weight sequence, $\phi:[0,1] \rightarrow[0,1]$ induces an endomorphism $T$ of $D([0,1], M), x_{0}$ is a fixed point of $\phi$ and $f$ is an eigenfunction of $T$ with eigenvalue $\lambda$. If $\lambda \neq 0,1$, and if $\lambda \neq\left(\phi^{\prime}\left(x_{0}\right)\right)^{n}$ for all positive integers $n$, then $f^{(m)}\left(x_{0}\right)=0$ for all non-negative integers $m$.

Proof: Let $\lambda$ and $f$ satisfy the hypothesis. Since $f$ is an eigenfunction, $\lambda f(x)=f(\phi(x))$, and since $\lambda \neq 1$, we have $f\left(x_{0}\right)=0$.

Now suppose that $m \geq 1$ is given, and for $\nu<m, f^{(\nu)}\left(x_{0}\right)=0$. We show that $f^{(m)}\left(x_{0}\right)=0$. For each positive integer $m, \lambda f^{(m)}(x)=$ $f^{(m)}(\phi(x))\left(\phi^{\prime}(x)\right)^{m}+\left[\left(\right.\right.$ terms containing sums and products of $f^{(\nu)}$ and powers of $\phi^{(\nu)}, \nu<m$.)] Since $f^{(\nu)}\left(x_{0}\right)=0$ for $\nu<m$, evaluating at $x_{0}$, we have $\lambda f^{(m)}\left(x_{0}\right)=f^{(m)}\left(x_{0}\right)\left(\phi^{\prime}\left(x_{0}\right)\right)^{m}$, and since $\lambda \neq\left(\phi^{\prime}\left(x_{0}\right)\right)^{m}$, we conclude that $f^{(m)}\left(x_{0}\right)=0$. Thus $f^{(n)}\left(x_{0}\right)=0$ for all non-negative integers $n$.

Lemma 2.3: Suppose that $\phi$ induces a compact endomorphism of $D([0,1], M)$, and that $x_{0}$ is the unique fixed point of $\phi$. Let $\lambda$ be a non-zero complex number. Suppose that $f \in D([0,1], M)$ is such that $f(\phi(x))=$ $\lambda f(x)$ and satisfies $f^{(k)}\left(x_{0}\right)=0$ for $k=0,1,2, \cdots$. Then $f$ must be the zero function. In particular, the endomorphism induced by $\phi$ has no non-zero eigenvalues other than $\phi^{\prime}\left(x_{0}\right)^{n}, n=0,1,2, \cdots$.

Proof: Suppose that $\lambda \neq 0$ and $f \in D([0,1], M)$ with $f(\phi(x))=\lambda f(x)$ and $f^{(k)}\left(x_{0}\right)=0$ for all $k=0,1,2, \ldots$. Since $\left|\phi^{\prime}\left(x_{0}\right)\right|<1$, we can choose $\alpha$ 
with $\left|\phi^{\prime}\left(x_{0}\right)\right|<\alpha<1$. Then there exists $n_{0} \in \mathbf{N}$ such that $\phi_{n_{0}}$ (and hence all later iterates of $\phi$ ) maps $[0,1]$ into a connected neighborhood $\mathcal{U}$ of $x_{0}$ with $\left|\phi^{\prime}(t)\right|<\alpha$ for all $t \in \mathcal{U}$. It then follows that there exists $C>0$ such that $\left|\phi_{n}(x)-x_{0}\right|<\alpha^{n-n_{0}}<C \alpha^{n}$ for all $x \in(0,1)$. Next choose $m \in \mathbf{N}$ large enough that $\alpha^{m}<|\lambda|$. This number $m$ will remain fixed for the remainder of this proof. Applying Taylor's theorem to the real and imaginary parts of $f$ we see that there is a constant $A>0$ such that $|f(x)| \leq A\left|x-x_{0}\right|^{m}$ for all $x \in[0,1]$. Since $f \circ \phi=\lambda f$, we have (for $\lambda \neq 0$ ), that $f(x)=f\left(\phi_{n}(x)\right) / \lambda^{n}$ for all $x \in[0,1]$ and all $n \in \mathbf{N}$. Thus, for such $x$ and $n$, we have

$|f(x)|=\left|f\left(\phi_{n}(x)\right) / \lambda^{n}\right| \leq A\left|\phi_{n}(x)-x_{0}\right|^{m} /|\lambda|^{n} \leq A C\left(\alpha^{n}\right)^{m} /|\lambda|^{n}=A C\left(\alpha^{m} /|\lambda|\right)^{n}$.

Letting $n$ tend to infinity gives us $f(x)=0$, as required. The last part of the conclusion now follows from Lemma 2.2 .

Theorem 2.4: Suppose that $\left(M_{n}\right)$ is a weight sequence, $\phi$ induces a compact endomorphism $T$ of $D([0,1], M)$ and $x_{0}$ is the unique fixed point of $\phi$. Then the spectrum $\sigma(T)=\left\{\left(\phi^{\prime}\left(x_{0}\right)\right)^{n}: n\right.$ is a positive integer $\} \cup\{0,1\}$.

Proof: Since $T$ is compact, $0 \in \sigma(T)$, and from Theorem 1.1, $\left|\phi^{\prime}\left(x_{0}\right)\right|<$ 1. Also Lemma 2.1 shows that $\sigma(T) \supset\left\{\left(\phi^{\prime}\left(x_{0}\right)\right)^{n}: n\right.$ is a positive integer $\} \cup$ $\{0,1\}$. On the other hand since $T$ is compact every non-zero element of $\sigma(T)$ is an eigenvalue. From this and Lemma 2.3 it follows that $\sigma(T) \subset\left\{\left(\phi^{\prime}\left(x_{0}\right)\right)^{n}\right.$ : $n$ is a positive integer $\} \cup\{0,1\}$, and thus equality holds.

We conclude this part by showing that the multiplicity of every non-zero eigenvalue is 1 .

Theorem 2.5: Suppose that $\left(M_{n}\right)$ is a weight sequence and that $\phi$ induces a compact endomorphism $T$ of $D([0,1], M)$. Then every non-zero eigenvalue of $T$ has multiplicity 1 .

Proof: We start by showing that the eigenvalue 1 has multiplicity 1 . Certainly $f(x) \equiv 1$ is an eigenvector. Next suppose that $g \in D([0,1], M)$ is another eigenvector. Then $g(\phi(x))=g(x)$ for all $x \in[0,1]$. It follows easily that $g\left(\phi_{n}(x)\right)=g(x)$ for all $x \in[0,1]$. However, since the induced endomorphism is compact, $\phi_{n}(x)$ converges to the unique fixed point, $x_{0}$, of $\phi$. Hence $g(x) \equiv g\left(x_{0}\right)$ as claimed.

For the remainder of the proof we may assume that $\phi^{\prime}\left(x_{0}\right) \neq 0$. Next suppose that $p$ is a positive integer. We show that the eigenvalue $\phi^{\prime}\left(x_{0}\right)^{p}$ also has multiplicity 1 . We recall that $\left|\phi^{\prime}\left(x_{0}\right)\right|<1$.

We first show that if $f \in D([0,1], M)$ with $f \circ \phi=\left(\phi^{\prime}\left(x_{0}\right)\right)^{p} f$, and if $f^{(p)}\left(x_{0}\right)=0$, then $f \equiv 0$. To this end assume that $f(\phi(x))=\left(\phi^{\prime}\left(x_{0}\right)\right)^{p} f(x)$ for all $x$. Then it is easy to see that $f^{(\nu)}\left(x_{0}\right)=0$ for $\nu=0,1, \cdots, p-1$. Also 
when we assume that $f^{(p)}\left(x_{0}\right)=0$, it is easy to see that $f^{(\nu)}\left(x_{0}\right)=0$ for $\nu>p$. Hence by Lemma 2.3, if $f \in D[0,1], M)$ and $f \circ \phi=\left(\phi^{\prime}\left(x_{0}\right)\right)^{p} f$ and $f^{(p)}\left(x_{0}\right)=0$, then $f \equiv 0$.

Finally suppose that $f, g \in D([0,1], M)$ and that both $f$ and $g$ are nonzero eigenvectors for $\phi^{\prime}\left(x_{0}\right)^{p}$. For $f$ and $g$ to be non-zero, both $f^{(p)}\left(x_{0}\right) \neq 0$ and $g^{(p)}\left(x_{0}\right) \neq 0$. Then the function $F$ defined by $F(x)=f(x)-\frac{f^{(p)}\left(x_{0}\right)}{g^{(p)}\left(x_{0}\right)} g(x)$ is an eigenvector for $\phi^{\prime}\left(x_{0}\right)^{p}$ and satisfies $F^{(p)}\left(x_{0}\right)=0$. Hence $F \equiv 0$ showing that $f$ and $g$ are linearly dependent.

\section{Part III. General endomorphisms}

As noted in the introduction (Theorem A), the calculations from [7] show that if $\left(M_{n}\right)$ is a non-analytic sequence, then every analytic selfmap $\phi$ of $[0,1]$ with $\left\|\phi^{\prime}\right\|_{\infty}<1$ induces a (compact) endomorphism of $D([0,1], M)$. Also (Theorem $\mathrm{A}^{\prime}$ ), if

$$
\frac{M_{m}}{m !} \frac{n !}{M_{n}} \leq \frac{B}{m^{n-m}}
$$

for $m \leq n$, and $\frac{\left\|\phi^{(k)}\right\|_{\infty}}{k !}$ is bounded, then $\left\|\phi^{\prime}\right\|_{\infty} \leq 1$ is sufficient for $\phi$ to induce an endomorphism.

We now relax the conditions on both $\left(M_{n}\right)$ and $\phi$ to prove a stronger version of Theorem $\mathrm{A}^{\prime}$. The new condition on $\left(M_{n}\right)$ can be shown to be equivalent to $\sup _{n} \frac{n^{2} M_{n-1}}{M_{n}}<\infty$, an easier condition to verify than $\frac{M_{m}}{m !} \frac{n !}{M_{n}} \leq$ $\frac{B}{m^{n-m}}$. In addition, $\phi$ can be assumed to be analytic, rather than satisfy the stronger condition $\sup _{k} \frac{\left\|\phi^{(k)}\right\|_{\infty}}{k !}<\infty$.

We begin with two easily checked observations.

Observation 1: If $\left(M_{n}\right)$ is a weight sequence, then $\sup _{n} \frac{n^{2} M_{n-1}}{M_{n}}<\infty$ if, and only if, there exist positive constants $B$ and $D$ such that whenever $m \leq n$ we have

$$
\frac{M_{m}}{m !} \frac{n !}{M_{n}} \leq B\left(\frac{D}{m}\right)^{n-m} .
$$

Observation 2: If $\left(M_{n}\right)$ is a weight sequence and $a_{j}$ are non-negative integers with $a_{1}+2 a_{2}+\cdots+n a_{n}=n$, then

$$
\prod_{k=1}^{n}\left(\frac{M_{k}}{k !}\right)^{a_{k}} \leq \frac{M_{n}}{n !} .
$$


Theorem 3.1: Suppose that $\left(M_{n}\right)$ is a non-analytic weight sequence satisfying $\sup _{n} \frac{n^{2} M_{n-1}}{M_{n}}<\infty$ and that $\phi$ is a analytic selfmap of $[0,1]$. If $\left\|\phi^{\prime}\right\|_{\infty} \leq 1$, then $\phi$ induces an endomorphism of $D([0,1], M)$.

Proof: As noted, if $\sup _{n} \frac{n^{2} M_{n-1}}{M_{n}}<\infty$, then there exist positive constants $B$ and $D$ such that $\frac{M_{m}}{m !} \frac{n !}{M_{n}} \leq B\left(\frac{D}{m}\right)^{n-m}, m \leq n$. Suppose $\phi$ satisfies the hypotheses with $\left\|\phi^{\prime}\right\|_{\infty} \leq 1$. Let $F \in D([0,1], M)$. We show that $F \circ \phi \in D([0,1], M)$.

The following equality for higher derivatives of composite functions is known as Faà di Bruno's formula.

$$
\frac{d^{n}}{d x^{n}}(F \circ \phi)=\sum_{m=0}^{n} F^{(m)}(\phi)\left(\sum_{a} \frac{n !}{a_{1} ! a_{2} ! \cdots a_{n} !} \prod_{k=1}^{n}\left(\frac{\phi^{(k)}}{k !}\right)^{a_{k}}\right)
$$

where the inner sum $\sum_{a}$ is over non-negative integers $a_{1}, a_{2}, \cdots, a_{n}$ satisfying $a_{1}+a_{2}+\cdots+a_{n}=m$ and $a_{1}+2 a_{2}+\cdots+n a_{n}=n$.

Throughout the proof the inner sum $\sum_{a}$ will always be over non-negative integers $a_{1}, a_{2}, \cdots, a_{n}$ satisfying $a_{1}+a_{2}+\cdots+a_{n}=m$ and $a_{1}+2 a_{2}+\cdots+n a_{n}=n$.

Thus, Faà di Bruno's formula implies that

$$
\left\|\frac{d^{n}}{d x^{n}}(F \circ \phi)\right\|_{\infty} \leq \sum_{m=0}^{n}\left\|F^{(m)}(\phi)\right\|_{\infty}\left(\sum_{a} \frac{n !}{a_{1} ! a_{2} ! \cdots a_{n} !} \prod_{k=1}^{n}\left(\frac{\left\|\phi^{(k)}\right\|_{\infty}}{k !}\right)^{a_{k}}\right)
$$

and so

$$
\sum_{n=0}^{\infty} \frac{1}{M_{n}}\left\|\frac{d^{n}}{d x^{n}}(F \circ \phi)\right\|_{\infty} \leq \sum_{n=0}^{\infty} \frac{1}{M_{n}} \sum_{m=0}^{n}\left\|F^{(m)}\right\|_{\infty}\left(\sum_{a} \frac{n !}{a_{1} ! a_{2} ! \cdots a_{n} !} \prod_{k=1}^{n}\left(\frac{\left\|\phi^{(k)}\right\|_{\infty}}{k !}\right)^{a_{k}}\right) .
$$

Then, after interchanging the order of summation, we have

$$
\sum_{n=0}^{\infty} \frac{1}{M_{n}}\left\|\frac{d^{n}}{d x^{n}}(F \circ \phi)\right\|_{\infty} \leq \sum_{m=0}^{\infty}\left\|F^{(m)}\right\|_{\infty} \sum_{n=m}^{\infty} \frac{1}{M_{n}}\left(\sum_{a} \frac{n !}{a_{1} ! a_{2} ! \cdots a_{n} !} \prod_{k=1}^{n}\left(\frac{\left\|\phi^{(k)}\right\|_{\infty}}{k !}\right)^{a_{k}}\right) .
$$

Since $\phi$ is analytic, there is some $C>0$ such that $\frac{\left\|\phi^{(k)}\right\|_{\infty}}{k !} \leq C^{k}$ for all $k$. We then have (denoting the greatest integer less than or equal to $x$ by $[x]$ ) that $\sum_{n=0}^{\infty} \frac{1}{M_{n}}\left\|(F \circ \phi)^{(n)}\right\|_{\infty} \leq \mathcal{A}_{1}+\mathcal{A}_{2}$ where 


$$
\mathcal{A}_{1}=\sum_{m=0}^{[C D]}\left\|F^{(m)}\right\|_{\infty} \sum_{n=m}^{\infty} \frac{1}{M_{n}}\left(\sum_{a} \frac{n !}{a_{1} ! a_{2} ! \cdots a_{n} !} \prod_{k=1}^{n}\left(\frac{\left\|\phi^{(k)}\right\|_{\infty}}{k !}\right)^{a_{k}}\right)
$$

and

$$
\mathcal{A}_{2}=\sum_{m=[C D]+1}^{\infty}\left\|F^{(m)}\right\|_{\infty} \sum_{n=m}^{\infty} \frac{1}{M_{n}}\left(\sum_{a} \frac{n !}{a_{1} ! a_{2} ! \cdots a_{n} !} \prod_{k=1}^{n}\left(\frac{\left\|\phi^{(k)}\right\|_{\infty}}{k !}\right)^{a_{k}}\right) .
$$

First we estimate $\mathcal{A}_{1}$. Recalling that, for the non-negative integers $a_{k}$ under consideration,

$$
\prod_{k=1}^{n}\left(\frac{M_{k}}{k !}\right)^{a_{k}} \leq \frac{M_{n}}{n !}
$$

we see that, for each $m$, the coefficient of $\left\|F^{(m)}\right\|_{\infty}$ is

$$
\begin{aligned}
& \sum_{n=m}^{\infty} \frac{1}{M_{n}}\left(\sum_{a} \frac{n !}{a_{1} ! a_{2} ! \cdots a_{n} !} \prod_{k=1}^{n}\left(\frac{\left\|\phi^{(k)}\right\|_{\infty}}{k !}\right)^{a_{k}}\right) \\
= & \sum_{n=m}^{\infty} \frac{1}{n !}\left(\sum_{a} \frac{n !}{a_{1} ! a_{2} ! \cdots a_{n} !} \frac{n !}{M_{n}} \prod_{k=1}^{n}\left(\frac{M_{k}}{k !} \frac{\left\|\phi^{(k)}\right\|_{\infty}}{M_{k}}\right)^{a_{k}}\right) \\
\leq & \sum_{n=m}^{\infty} \frac{1}{n !}\left(\sum_{a} \frac{n !}{a_{1} ! a_{2} ! \cdots a_{n} !} \prod_{k=1}^{n}\left(\frac{\left\|\phi^{(k)}\right\|_{\infty}}{M_{k}}\right)^{a_{k}}\right) .
\end{aligned}
$$

Now from formula B3, page 823 in [1], the right hand side of the last inequality equals $\frac{1}{m !}\left(\sum_{k=1}^{\infty} \frac{\left\|\phi^{(k)}\right\|_{\infty}}{M_{k}}\right)^{m}=\frac{1}{m !}\left(\|\phi\|_{D}-\|\phi\|_{\infty}\right)^{m}$.

Therefore,

$$
\begin{gathered}
\mathcal{A}_{1}=\sum_{m=0}^{[C D]}\left\|F^{(m)}\right\|_{\infty} \sum_{n=m}^{\infty} \frac{1}{M_{n}}\left(\sum_{a} \frac{n !}{a_{1} ! a_{2} ! \cdots a_{n} !} \prod_{k=1}^{n}\left(\frac{\left\|\phi^{(k)}\right\|_{\infty}}{k !}\right)^{a_{k}}\right) \\
\leq \sum_{m=0}^{[C D]} \frac{\left\|F^{(m)}\right\|_{\infty}}{m !}\left(\|\phi\|_{D}-\|\phi\|_{\infty}\right)^{m}
\end{gathered}
$$

which is finite. 

have

We next show that $\mathcal{A}_{2}$ is finite. Given that $\frac{n !}{M_{n}} \frac{M_{m}}{m !} \leq B\left(\frac{D}{m}\right)^{n-m}$, we

$$
\begin{gathered}
\mathcal{A}_{2}=\sum_{m=[C D]+1}^{\infty}\left\|F^{(m)}\right\|_{\infty} \sum_{n=m}^{\infty} \frac{1}{M_{n}}\left(\sum_{a} \frac{n !}{a_{1} ! a_{2} ! \cdots a_{n} !} \prod_{k=1}^{n}\left(\frac{\left\|\phi^{(k)}\right\|_{\infty}}{k !}\right)^{a_{k}}\right) \\
=\sum_{[C D]+1}^{\infty} \frac{\left\|F^{(m)}\right\|_{\infty}}{M_{m}} m ! \sum_{n=m}^{\infty} \frac{1}{n !}\left(\sum_{a} \frac{n !}{M_{n}} \frac{M_{m}}{m !} \frac{n !}{a_{1} ! a_{2} ! \cdots a_{n} !} \prod_{k=1}^{n}\left(\frac{\left\|\phi^{(k)}\right\|_{\infty}}{k !}\right)^{a_{k}}\right) \\
\leq B \sum_{[C D]+1}^{\infty} \frac{\left\|F^{(m)}\right\|_{\infty}}{M_{m}} m ! \sum_{n=m}^{\infty} \frac{1}{n !}\left(\sum_{a} \frac{n !}{a_{1} ! a_{2} ! \cdots a_{n} !} \prod_{k=1}^{n}\left(\frac{\left\|\phi^{(k)}\right\|_{\infty} D^{k-1}}{k ! m^{k-1}}\right)^{a_{k}}\right) \\
=B \sum_{[C D]+1}^{\infty} \frac{\left\|F^{(m)}\right\|_{\infty}}{M_{m}}\left(\sum_{k=1}^{\infty} \frac{\left\|\phi^{(k)}\right\|_{\infty} D^{k-1}}{k ! m^{k-1}}\right)^{m},
\end{gathered}
$$

where we have again used the previously quoted formula from [1]. Therefore (assuming, as we may, that $\phi$ is non-constant),

$$
\begin{aligned}
\mathcal{A}_{2} \leq B \sum_{[C D]+1}^{\infty} \frac{\left\|F^{(m)}\right\|_{\infty}}{M_{m}}\left\|\phi^{\prime}\right\|_{\infty}^{m}\left(1+\sum_{k=2}^{\infty} \frac{\left\|\phi^{(k)}\right\|_{\infty} D^{k-1}}{k !\left\|\phi^{\prime}\right\|_{\infty} m^{k-1}}\right)^{m} \\
=B \sum_{[C D]+1}^{\infty} \frac{\left\|F^{(m)}\right\|_{\infty}}{M_{m}}\left\|\phi^{\prime}\right\|_{\infty}^{m}\left(1+\sum_{k=2}^{\infty} \frac{C^{k} D^{k-1}}{\left\|\phi^{\prime}\right\|_{\infty} m^{k-1}}\right)^{m} \\
=B \sum_{[C D]+1}^{\infty} \frac{\left\|F^{(m)}\right\|_{\infty}}{M_{m}}\left\|\phi^{\prime}\right\|_{\infty}^{m}\left(1+\frac{C}{\left\|\phi^{\prime}\right\|_{\infty}} \sum_{k=2}^{\infty} \frac{C^{k-1} D^{k-1}}{m^{k-1}}\right)^{m} \\
=B \sum_{[C D]+1}^{\infty} \frac{\left\|F^{(m)}\right\|_{\infty}}{M_{m}}\left\|\phi^{\prime}\right\|_{\infty}^{m}\left(1+\frac{C^{2} D}{\left\|\phi^{\prime}\right\|_{\infty}} \frac{1}{m-C D}\right)^{m} .
\end{aligned}
$$

Since $\left(1+\frac{C^{2} D}{\left\|\phi^{\prime}\right\|_{\infty}} \frac{1}{m-C D}\right)^{m}$ is easily seen to be bounded in $m$, we conclude that $\mathcal{A}_{2}$ is finite when $\left\|\phi^{\prime}\right\|_{\infty} \leq 1$, as claimed.

Most non-analytic weight sequences whose growth is, in some sense, regular and which do not satisfy the conditions of Theorem 3.1 are such that $n^{2} M_{n} / M_{n+1} \rightarrow \infty$ as $n \rightarrow \infty$. In this case $\left\|\phi^{\prime}\right\|_{\infty} \leq 1$ is no longer sufficient for $\phi$ to induce an endomorphism of $D([0,1], M)$ as the following theorem shows. 
Theorem 3.2: Let $\left(M_{n}\right)$ be a weight sequence such that $n^{2} M_{n} / M_{n+1} \rightarrow$ $\infty$ as $n \rightarrow \infty$. Then the map $\phi(x)=\left(1+x^{2}\right) / 2$ does not induce an endomorphism of $D([0,1], M)$.

Proof: Consider the functions $F_{A}(t)=\exp (A(t-1))$ where $A$ varies through the positive real numbers. Clearly when $t=1$, all the derivatives involved are non-negative.

Then for $n \geq 2$, the second term $(m=n-1)$ of the Faà di Bruno expansion of $\left(F_{A} \circ \phi\right)^{(n)}(1)$ tells us that

$$
\begin{gathered}
\left(F_{A} \circ \phi\right)^{(n)}(1) \geq\left(\begin{array}{l}
n \\
2
\end{array}\right) F_{A}^{(n-1)}(\phi(1))\left(\phi^{\prime}(1)\right)^{n-2} \phi^{\prime \prime}(1) \\
=\left(\begin{array}{l}
n \\
2
\end{array}\right) F_{A}^{(n-1)}(1)=\left(\begin{array}{l}
n \\
2
\end{array}\right) A^{n-1} .
\end{gathered}
$$

Set $C=1 / 4$. We obtain, for $n \geq 2$, and such functions $F_{A},\left\|\left(F_{A} \circ \phi\right)^{(n)}\right\|_{\infty} \geq$ $C n^{2}\left\|F_{A}^{(n-1)}\right\|_{\infty}$.

Let $B>0$. Choose $N \in \mathbf{N}$ with $N>2$ and such that, for all $k \geq N$ we have $k^{2} M_{k-1} / M_{k}>B$. Choose $A>0$ such that $\sum_{k=0}^{N-1} A^{k} / M_{k}<(1 / 2) A^{N} / M_{N}$. Then, for the function $F_{A}$ we have

$$
\begin{gathered}
\sum_{k=N}^{\infty}\left\|F_{A}^{(k)}\right\|_{\infty} / M_{k}=\sum_{k=N}^{\infty} A^{k} / M_{k} \\
\geq(1 / 2)\left\|F_{A}\right\|_{D} .
\end{gathered}
$$

But, by the above, we also have

$$
\begin{gathered}
\left\|F_{A} \circ \phi\right\|_{D} \geq \sum_{k=N}^{\infty}\left\|\left(F_{A} \circ \phi\right)^{(k)}\right\|_{\infty} / M_{k} \\
\geq \sum_{k=N}^{\infty} C k^{2}\left\|F_{A}^{(k-1)}\right\|_{\infty} / M_{k}=C \sum_{k=N}^{\infty} k^{2} A^{k-1} / M_{k} \\
=C \sum_{k=N}^{\infty} k^{2}\left(M_{k-1} / M_{k}\right)\left(A^{k-1} / M_{k-1}\right) \geq C B \sum_{k=N}^{\infty} A^{k-1} / M_{k-1} \\
\geq(C / 2) B\left\|F_{A}\right\|_{D} .
\end{gathered}
$$

As $B>0$ was arbitrary, this shows that $\phi$ cannot induce a bounded endomorphism of $D([0,1], M)$ and so $\phi$ does not induce an endomorphism at all. 
The rate of growth of $\frac{n^{2} M_{n}}{M_{n+1}}$ and the order of the entire function $g(z)=$ $\sum_{n=0}^{\infty} \frac{z^{n}}{M_{n}}$ are somewhat related. In particular, if $\frac{n^{2} M_{n}}{M_{n+1}}$ is bounded, then the order $\rho$ of $g$ is less than $\frac{1}{2}$, or $\rho=\frac{1}{2}$ and the type $\tau$ is finite. On the other hand, if $\lim _{n \rightarrow \infty} \frac{n^{2} M_{n}}{M_{n+1}}=\infty$, then $\rho>\frac{1}{2}$, or $\rho=\frac{1}{2}$ and $\tau=\infty$. Using order and type of the function $g(z)$, one can also show the following.

Proposition 3.3: Let $\left(M_{n}\right)$ be a non-analytic weight function. Suppose that $\phi:[0,1] \rightarrow[0,1]$ is in $D([0,1], M)$ with $\phi(1)=1=\phi^{\prime}(1)=\left\|\phi^{\prime}\right\|_{\infty}$, and $\phi^{(\nu)}(1) \geq 0$ for all $\nu$. Let $g(z)=\sum_{n=0}^{\infty} \frac{z^{n}}{M_{n}}$, an entire function with order $\rho$ and type $\tau$. If $\phi^{\prime \prime}(1) \neq 0$ and $\rho>\frac{1}{2}$, then $\phi$ does not induce an endomorphism of $D([0,1], M)$. Also, if $\rho=\frac{1}{2}$ and $\tau=\infty$, then such $\phi$ does not induce an endomorphism.

\section{References}

[1] M. Abramowitz and I. Stegun, Handbook of Mathematical Functions with Formulas, Graphs, and Mathematical Tables, U.S. Department of Commerce, Washington, D.C., 1964.

[2] R. P. Boas, Entire Functions, Academic Press, New York, 1954.

[3] H. G. Dales, Banach Algebras and Automatic Continuity, LMS Monographs 24, Clarenden Press, Oxford, 2000.

[4] H. G. Dales and A. M. Davie, Quasianalytic Banach function algebras, J. Funct. Anal. 13 (1973), 28-50.

[5] J. F. Feinstein and H. Kamowitz Endomorphisms of algebras of infinitely differentiable functions on compact plane sets, J. Funct. Anal. 173 (2000), 61-73.

[6] H. Kamowitz, Compact endomorphisms of Banach algebras, Pac. J. Math. 89 (1980), 313-325.

[7] H. Kamowitz Endomorphisms of Banach algebras of infinitely differentiable functions, Proceedings of the 13th International Conference on Banach Algebras in Blaubeuren, Germany, Walter de Gruyter, Berlin, New York 1998. 
[8] J. K. Langley Proof of a conjecture of Hayman concerning $f$ and $f^{\prime \prime}$, J. London Math. Soc. (2) 48 (1993), 500-514.

\author{
School of Mathematical Sciences \\ University of Nottingham \\ Nottingham NG7 2RD, England \\ email: Joel.Feinstein@nottingham.ac.uk \\ and \\ Department of Mathematics \\ University of Massachusetts at Boston \\ 100 Morrissey Boulevard \\ Boston, MA 02125-3393 \\ email: hkamo@cs.umb.edu
}

2000 Mathematics Subject Classification: 46J15, 47B48

This research was supported by EPSRC grants GR/M31132 and GR/R09589 